\title{
Control of Malaria by Blocking Transmission of Plasmodium
}

\author{
Johnson Adejoh ${ }^{1}$, Maxwell O. Egua ${ }^{2} \&$ Michael P. Okoh ${ }^{1}$ \\ ${ }^{1}$ Department of Medical Biochemistry, Faculty of Basic Medical Sciences, College of Health Sciences, University \\ of Abuja, P.M.B 117 FCT, Abuja, Nigeria \\ ${ }^{2}$ Department of Pharmacology and Therapeutics, Faculty of Basic Clinical Sciences, College of Health Sciences, \\ University of Abuja, P.M.B 117 FCT, Abuja, Nigeria \\ Corresponding author: Michael P. Okoh, Department of Medical Biochemistry, Faculty of Basic Medical Sciences, \\ College of Health Sciences, University of Abuja, P.M.B 117 FCT, Abuja, Nigeria. Tel: 234-703-668-3068. E-mail: \\ Michael.okoh@uniabuja.edu,ng
}

Received: August 8, 2018

Accepted: August 21, 2018

Online Published: September 25, 2018

doi:10.5539/ijb.v10n4p29

URL: https://doi.org/10.5539/ijb.v10n4p29

\begin{abstract}
Malaria is a major global health problem with the greatest burden in sub-Saharan Africa (sSA). Unfortunately, Nigeria accounts for 25 percent of the world's malaria burden and it accounts for more deaths than HIV/AIDS. The causative agent of malaria is plasmodium species. This paper reviews the current approaches to inhibiting plasmodium transmission, and the phyto active compound currently in use in the sSA (particularly in Nigeria) with the goal to ameliorate the high incidence of malaria and to correlating it with recent progress and scientific understanding.

Using search engines, several databases including Google scholar, Pub Med, Academic Resource Index, Scopus, etcetera, were utilized to source for relevant publications and literatures.

The complex life cycle of the Plasmodium species (causative agent of malaria) gives room for measures that can disrupt its completion. Several methods are currently being tested and experimented on to disrupt the parasite transmission. The disruption of a cell surface transport protein, Feline Leukemia Virus subgroup C Receptor (FLVCR) that pumps heme out of the cell; Gene silencing-techniques used to reduce the levels of FLVCR in the mosquito gut; Prevention of the interaction between the plasmodium TRAP and the Anopheles Saglin protein, which aid the malaria parasite invasion of the mosquito salivary gland; Prevention of the Interaction of Surface Enolase and Plasminogen of Mammalian Blood, disrupting an important role in ookinete invasion of the mosquito midgut; the use of Plants with antimicrobial peptides(cyclotide), that possess structural similarities to SM1 peptide, an inhibitor of plasmodium TRAP-saglin binding;and Use of Phyto-Active Compounds to Block Plasmodium Transmission. These approaches are novel methods in the control and transmission of plasmodium species/malaria.

Chemically, phytochemicals with structural similarities to artemisinin, (asesquiteterpene lactone containing an unusual peroxide bridge) is thought of to be present in certain plants with antimalarial and other medicinal value.
\end{abstract}

Keywords: Phytochemicals, Gene silencing, Plasmodium, Natural competitors, Plants

\section{Introduction}

The effects of malaria disease in Nigeria and other sub Saharan African (sSA) countries cannot be over look. Due to the frequency of malaria it necessitates more than 1.0 million hospital admissions with high economic burdens, which is estimated at over $\$ 1.0$ Billion U. S. Dollars (USD) of healthcare expenditures (World Health Organization [WHO], 2017), causing a reduction in the population and certain socio-economic factors.

Plasmodium species, the causative agent of malaria, is responsible for about 500 million clinical cases and about 2 million deaths every year, mostly of African children (Snow, Guerra, Noor, Myint, \& Hay, 2005), and the number of cases has increased over the last ten-fifteen years (Breman, Egan, \& Keusch, 2001) indicating that the available therapeutic agents to combat the disease are inadequate. Malaria, unlike other two major infectious diseases (Acquired Immunodeficiency Syndrome (AIDS) and tuberculosis), depends on an intermediate insect vector for transmission to occur. 
There is a complex life cycle of the causative agent (Plasmodiumspecies). This starts with the insect vector, the female mosquito, feeding on the blood of infected individual ingesting gametocytes. After ingestion, gametocytes differentiate into male and female gametes that mate to produce zygotes within the mosquito midgut; differentiating into motile ookinetes that invade the midgut epithelium. After emerging on the hemocoel side, ookinetes differentiate into sessile oocysts that take between one-two weeks to mature (depending on the Plasmodium species), releasing thousands of sporozoites into the open hemolymph circulation of the mosquito. Of all mosquito tissues, the sporozoitesin the hemolymphpreferentially invade the mosquito salivary gland (Ghosh, Devenport, Jethwaney, Kalume, \& Pandey, 2009). Several lines of evidence suggest that sporozoite invasion of the salivary gland requires specific receptor-ligand interactions. For instance, $P$. Knowlesisporozoites can invade the salivary glands of Anopheles dirus but not salivary glands of Anopheles freeborni (Rosenberg, 1985). Inhibition experiments with polyclonal serum against whole Aedesaegypti salivary glands and against specific salivary gland epitopes all suggest the involvement of specific receptors (Korochkina, Barreau, Pradel, Jeffery, \& Li, 2006). Recently a monoclonal antibody directed against Anopheles gambiae salivary glands was shown to recognize a glycosylated homodimer protein made up of $50 \mathrm{kDa}$ subunits, designated saglin (Ghoshet al., 2009). The transmission cycle is completed when an infected mosquito bites another individual releasing some of its sporozoites (infecting process). The complex life cycle of the parasite in the mosquito constitutes a potential weak link that is being exploited for malaria control.

Interference with the transmission cycle of plasmodium parasite from mosquito to the host has recently become a target point for the control of malaria disease (Smith, Vega-Rodriquez, \& Jacobs-Lorena, 2014). Furthermore, specialist says that blocking the transmission of parasite between the host and the mosquito may prove useful in the control of malaria disease (Arrow, 2004). Herein, we discuss below some strategies currently considered useful in truncating malaria life cycle.

In this review we bring into focus recent scientific advances in the attempt to understand the complex life cycle of mosquito malaria transmission. Also we relate plants with phytochemical properties currently used in sub-Saharan Africa (sSA) to mitigate the malaria scourge. And, bearing in mind, the links between medicinal plants and successful anti-malarial drug discovery, we postulate, that these plants with phyto activities may act via their cell-penetrating peptides (CPPs).

\section{Literature Review}

Several strategies are currently been thought of as a possible means to combat the causative pathogen (Plasmodium) as well as the vector (mosquito), which carries this pathogenic organism. The life cycle of the pathogenic organism has been a subject of interest in the design of antiplasmodial drugs. Novel design of cysteine protease inhibitor responsible for prevention of the formation of hemozoine in human erythrocyte has certain constraints due to the development of different strains of plasmodium parasite that expresses resistance to antimalarial drugs (Abdukadir, Ismail, Sani, Elewechi, \& Adamu, 2016). This has led researchers to focus on other ways, which may prove useful in the control of malaria, moving forward.

Thrombospondin-related adhesive protein (TRAP) (Akhouri, Sharma, Malhota, \& Amit, 2008) is a protein expressed in sporozoites and conserved in all Plasmodium species. TRAP is essential for sporozoite gliding, cell invasion and in vivo infectivity (Akhouri et al., 2008). This protein is localized to the parasite micronemes (Rogers, Malik, Mellouk, Nakamura, \& Rogers, M. D. et al., 1992) and becomes surface exposed at the sporozoite's anterior tip, particularly upon contact with the host (Gantt, Persson, Rose, Birkett, \& Abagyan, 2000). It is also released onto the substrate during gliding locomotion (Kappe, Bruderer, Gantt, Fujioka, \& Nussenzweig, 1999). The extracellular portion of TRAP contains two conserved adhesive domains: a von Willebrand factor A-domain ('A-domain') (Girma, Meyer, Verweij, Pannekoek, \& Sixma, 1987) and a thrombospondin type I repeat (TSR) (Lawler \& Hynes, 1986). This combination of TSR- and A-domains is also found in micronemaltransmembrane proteins of many apicomplexans.Independent lines of evidence suggest that these proteins have similar functions to TRAP (Templeton, Kaslow, \& Fidock, 2000). More recent results show that sporozoites carrying a mutated TRAP domain-A are impaired in salivary gland invasion but not in gliding motility suggesting that the two processes are functionally distinct and that the A-domain is involved in recognition and/or attachment to salivary gland receptor molecules (Matuschewski, Nunes, Nussenzweig, \& Menard, 2002).Using phage display library, a peptide, SM1, was identifiedto bind the outer surface of anopheline salivary glands and inhibits sporozoite invasion, most likely because the peptide binds to and competes for a putative sporozoite receptor on the surface of the salivary glands (Ghosh, Ribolla, \& Jacobs-Lorena, 2001). This is true both for the rodent $P$. berghei parasite (Ghoshet al., 2001) and for the human P. Falciparumparasite (based on the findings from the Laboratory of Malaria and Vector Research, National Institute of Allergy and Infectious Disease, Rockville, USA),suggesting that salivary gland invasion of the two parasite species have similar pathways. However, the precise identity of the 
putative receptor and of the sporozoite ligand remained unknown. The SM1 here is recognised as a peptide similar to the TRAP domain-A and that SM1 interacts specifically with saglin on the surface of the salivary gland (Ghoshet al., 2009). Additional evidence suggests that TRAP domain-A directly interacts with saglin and that this interaction is essential for sporozoite invasion of the salivary gland (Ghoshet al., 2009).

After the mosquito ingests an infected blood meal, male and female gametes mate in the midgutlumen giving rise to zygotes that differentiate into motile ookinetes. After crossing the peritrophic matrix aided by chitinase secretion (Tsai, Hayward, Langer, Fidock, \& Vinetz, 2001; Dessenet al., 2003), the ookinete establishes specific molecular interactions with the midgut epithelial cells followed by their invasion and traversal.Several proteins from the ookineteand the mosquito aminopeptidase 1 (APN1), annexin-like proteins, carboxypeptidase B, croquemort scavenger receptor homolog, and calreticulin (Dinglasanet al., 2007; Lavazecet al., 2007; Rodriguez-Mdelet al., 2007; Gonzalez-Lazaro, Dinglasan, Hernandez-Henandez, \& Rodriquez, 2009) have been suggested to be involved in this process.

Mosquito midgut invasion by Plasmodiumookinetes is considered to be a promising target for transmission-blocking intervention as parasite numbers undergo a major bottleneck at this stage (Sinden, 2010; Wang \& Jacob-Lorena, 2013). However, the only molecular interaction between the ookinete and the midgut characterized is the in vitro interaction between parasite Pvs 25 and mosquito calreticulin (Rodriguez-Mdelet al., 2007). Circumstantial evidence suggests that ookinete invasion of the mosquito midgut requires specific interactions between parasite and mosquito components (Ghoshet al., 2001; Dinglasan et al., 2007).

In an attempt to elucidate these interactions at the molecular level, a phage display library for peptides that bind to the Anophelesmidgut epithelium had been screened. This screening identified SM1, a dodecapeptide that binds to the midgut luminal surface and importantly, strongly inhibits Plasmodium bergheiookinete invasion (Ghosh et al., 2001). Midgut expression of the SM1 peptide by transgenic mosquitoes also inhibits P. Bergheiookinete invasion (Ito, Ghosh, Moreira, Wimmer, \& Jacobs-Lorena, 2001). Further work indicated that SM1 structurally mimics the ookinete surface protein enolase, which was hypothesized to be involved in the recognition of a midgut receptor (Ghosh, Coppens, Gardsvoll, Ploug, \& Jacobs-Lorena, 2011). A mosquito midgut surface protein, enolasebinding protein (EBP), that binds both SM1 and ookinete surface enolase, is required for midgut invasion. Another important peptide useful in plasmodium transmission is themidgut peptide 2 (MP2). This peptide binds to a putative alternate receptor and inhibits ookinetemidgut invasion of $P$. berghei (both SM1-sensitive and SM1-resistant) and Plasmodium falciparum, which has an important implication for the development and implementation of malaria transmission-blocking strategies.

\subsection{Disruption of Feline LeukemiaVirus SubgroupCReceptor (FLVCR)}

The disruption of a cell surface transport protein called Feline LeukemiaVirus SubgroupCReceptor (FLVCR) that pumps heme out of the cell is one basic target point for the prevention of transmission of Plasmodiumparasite between mosquito and the host organism. This surface protein is required for pro-erythroblast survival known to facilitate macrophage heme iron recycling (Yang et al., 2010).Although, the mechanistic process involved in thisheme export and the substrate specificity are yet to be fully understood. However, recent study using $[(55) \mathrm{Fe}]$ heme and the fluorescent heme with analog zinc mesoporphyrin suggested that FLVCR transport may be due to the avidity of extracellular heme-binding protein(hemopexin) (Yang et al., 2010), ashemopexin directly interacts with FLVCR enabling it to function in a unidirectional gradient-dependent transporter.

Hemopexin, also known as beta-1B-glycoprotein, is a protein that is encoded by HPX-gene in human (Altruda, Poli, Restagno, \& Silengo, 1988) and belongs to hemopexin family of proteins. Haemoglobin and its scavenger protein hemopexin (Hx) associate with High Density Lipoprotein (HDL), and influence the inflammatory properties of HDL; hence, HDL from Hx-null mice is pro-inflammatory (Mehta \& Reddy, 2016). Moreover, deficiency of hemopexin is thought to be associated with various other inflammatory diseases such as septic shock and experimental autoimmune encephalomyelitis (Mehta \& Reddy, 2016).This encoded protein, transport heme from the plasma to the liver and are thought to be involved in protecting cells from oxidative stress. Mutation inhemopexin or heme oxygenase-2 gene can aggravate brain injury followed by stroma-free haemoglobin-induced intra-cerebral haemorrhage (Bo Ma et al., 2016). Similarly, such mutation pattern exists in mosquito following a blood meal containing a mutated HPX-gene (Dana et al., 2005).

Understanding the molecular mechanism underpinning these processes in mosquito may be useful in attempt to preventing the transmission of plasmodium parasite. Disrupting vector parasite interactions froman essential part in these processes, preventing the ability of the sporozoites to migrate to the liver and establish a normal hepaticinfection. Hence, the recent characterization of the interaction between mosquito gamma interferon inducible thiolreductase (GILT), andPlasmodiumsporozoites (Schleicher et al., 2018), is a critical part in this 
whole process bearing, it could help unravel newpathways that may be associated with motility regulation of Plasmodium parasites and potentiate design of novel therapeutics to preventmalaria disease transmission.

\subsection{Reduction of FLVCR by Gene Silencing-Techniques}

Gene silencing-techniques had been used to reduce the levels of FLVCR in the mosquito gut thus serving as a means of blocking transmission of plasmodium in mosquito (Quigley, 2010).Gene silencing is the regulation of gene expression in a cell to prevent the expression of a certain gene (Redberry,2006). The mechanism can be achieved during transcription or translation (Redberry, 2006). Gene silencing as a method is being increasingly employed to produce therapeutics to combat cancer and other infectious diseases, including neurodegenative disorders.

Gene silencing is often considered the same as gene knockdown (Hood, 2004), bearing that both methods silence and reduce gene expression (Mocellin \& Provenzano, 2003). In contrast, when genes are knocked out, they are completely erased from the organism's genome and, thus, have no expression (Hood, 2004). Gene silencing/knockdown both utilises similar methods, such asRNA interference (RNAi), cluster regularly interspaced short palindromic repeats (CRISPR), or small interfering/silencing RNA(siRNA), which generally reduce the expression of a gene by at least $70 \%$ but do not completely eliminate it. Methods using gene silencing are often considered better than gene knockouts since they allow researchers to study essential genes that are required for the animal models to survive and cannot be removed. In addition, they provide a more complete view on the development of diseases since diseases are generally associated with genes that have a reduced expression (Hood, 2004).

The use of gene silencing-techniques poses a great promise by executing the reduction of the number of FLVCR1 gene (by knocking down) in the mosquito midgut thereby reducing the number of FLVCR available to bind to hemopexin (that enhances plasmodium parasite transmission into its salivary glands)(Arrow, 2004). Such disruption of the function of the protein by gene silencing could inhibit parasite transmission, with part of the protein potentially used as an antigen to try to stimulate a vaccine in the human host (Quigley, 2010).This vaccine is expected to produce antibody that would block FLVCR and increase oxidative stress resulting in the plasmodium incomplete life cycle, thereby preventing the spread of malaria.

\subsection{Prevention of the Interaction between Plasmodium Trap and the Anopheles Saglin Protein}

Prevention of the interaction between the plasmodium TRAP and the Anopheles Saglin protein, which aid the malaria parasite invasion of the mosquito salivary gland, can also be targeted in the hope of blocking plasmodium parasite transmission between mosquito and the human host. Saglin appears to be a secreted product of extracellular protein thus, whether it is partly present in the lumen of salivary glands and or in the saliva remains is to be determined, although some experimental evidence suggests the presence of saglin on the outer surface of the distal lobes of salivary gland, facing the hemocoel (Myung, Marshall, \& Sinnis, 2004). Sporozoite TRAP-domain-A interaction with salivary gland is mediated by binding to saglin, although the mechanism of this interaction remains unclear (Ghosh\& Jacobs-Lorena, 2011). Moreover, it is speculated that saglin present at the basal side of the salivary gland cells could interact with other membrane bound proteins on the basal salivary-gland surface and facilitates sporozoite interaction and salivary gland invasion (King et al., 2011).

The findings that the salivary gland and midgut peptide 1 (SM1 peptide) 'pulls-down' saglin from the salivary gland surface and anti-SM1 antibodies recognize TRAP on the sporozoites surface suggested that sporozoite TRAP protein mediates salivary glands invasion by binding to one of its components (Ghoshet al., 2009). Incubation of recombinant TRAP domain-A with salivary glands suggested that indeed the protein binds directly to its surface (Ghoshet al., 2009). It had been established that SM1 compete in a dose dependent manner with TRAP domain-A, at a concentration of $0.5 \mathrm{mM}$. Partial inhibition was observed at a higher concentration which effectively prevented TRAP domain-A binding (Ghoshet al., 2009). Moreover, in a complementary set of experiments, it was demonstrated that binding of TRAP domain-A to the salivary gland surface effectively prevents SM1 binding (Korochkinaet al., 2006). These experimental evidences therefore, showed that the SM1 peptide and TRAP domain-A competes for the same receptor on the salivary gland surface. These findings led to the concept that the two proteins may interact, a hypothesis that was further verified via in vitro and in vivo experiments (Snow et al., 2005), indicating that maybe, both $P$. falciparum and $P$. berghei use saglin as a receptor (Snow et al., 2005).

Mutational analysis of TRAP-saglin interactions indicates that TRAP domain-A contains a metal-ion-dependent adhesion site (MIDAS) motif that is essential for sporozoite invasion of salivary glands (Sultan, Thathy, Frevert, Robson, \& Crisanti, 1997). In particular, the mutation of the critical Thr126 amino acid to Ala, strongly impaired 
(80\%) sporozoite invasion of mosquito salivary glands (Matuschewskiet al., 2002). However, the molecular mechanism underlying this impairment is not fully understood.

Circumstantial evidence suggests that invasion process in the mosquito salivary glands vector is mediated by specific receptor-ligand interactions. Both TRAP and saglin proteins had independently been implicated in the process of invasion (Brennan, Kent, Dhar, Fujioka, \& Kumar, 2000; Matuschewskiet al., 2002). Saglin is expressed specifically on the salivary gland distal lobes, which is the region invaded by sporozoites (Brennan et al., 2000). The fact that the SM1 peptide blocks salivary gland invasion of both $P$. Berghei (Ghoshet al., 2001) and $P$. falciparum makes it likely that both parasite species use TRAP domain-A as a ligand for invasion. A search of available databases did not reveal any Plasmodium protein with similarity to the peptide, indicating that SM1 conformation, rather than primary amino acid sequence, is related to a sporozoite protein (Ghoshet al., 2009). SM1 binding to salivary glands was abolished by the reduction of the disulphide bond between two cysteine molecules (Yee, Shibata, Yun, Ron, \& Rapoport, 2004). An anti-SM1 antibody recognizes sporozoite TRAP thereby enhancing the TRAP domain-A, to bind the salivary glands. The binding is then competed for by the SM1 peptide. TRAP-related proteins mediate cell-cell and cell-matrix interactions in a wide variety of biological systems (Myunget al., 2004). Moreover, TRAP is an essential protein for gliding motility and sporozoite invasion of mosquito salivary glands (Yeeet al., 2004). Sporozoites harbouring mutations in TRAP domain-A (the part of the protein that resembles SM1) preserve gliding motility but are impaired in salivary gland invasion (Matuschewskiet al., 2002). Like TRAP, adhesive domains of CTRP (circumsporozoite-andTRAP-related protein) and WARP (ookinete extracellular matrix proteins) also mediate ookinete invasion of the mosquito midgut epithelial cells (González-Lázaro et al., 2009; Ghoshet al., 2011). TRAP domain-A harbours an important module termed MIDAS, within this module threonine (T126) and aspartate (D157) are conserved in all Plasmodium species (Kalume, Okulate, Zhong, Reddy \& Suresh, 2005). In a vertebrate model system, mutation of these residues to alanines prevent divalent cation( $\mathrm{Mg}^{2+}$ or $\mathrm{Mn}^{2+}$ ) coordination, and A-domain binding to its ligand, without affecting A-domain folding (Jethwaney, Lepore, Hassan, Mello, \& Rangarajan, 2005). It was reviewed that the T126-to-Ala mutation abrogates binding to salivary glands or to saglin in vitro, which facilitate the transmission of plasmodium parasite. These findings suggest that TRAP domain-A interaction with salivary glands is mediated by binding to saglin, as alignment of SM1 to the amino acid sequence of domain-A revealed that 6 out of 8 amino acids in the SM1 loop between the two cysteines loosely aligned within a region of the MIDAS domain (González-Lázaroet al., 2009; Ghoshet al., 2011). Benzo-phenonephotophore can be used to crosslink SM1 to the salivary gland protein(s) with which it interacts. The photo crosslinker has several advantages for the study of peptide-protein interactions which includes; (a) chemically more stable (than other photoactivatable reagents); (b) reacts preferentially with $\mathrm{C}-\mathrm{H}$ bonds (even in the presence of water); (c) compatibility with peptide synthesis methodology; and (d) broad (300-600 nm) action spectrum (Saviano, Improta, Benedetti, Carrozzini, \& Cascarano, 2004). Using SM1 linked peptide pull-down assays two candidate salivary gland proteins, saglin and an SG1-like protein have been identified (Ghoshet al., 2011). Saglin and the SG1-like protein share only $20 \%$ sequence similarity. SG1 belongs to a family of protein that encode a complex of high molecular weight proteins including a candidate receptor for malaria sporozoite invasion of salivary glands (Korochkinaet al., 2006). The possible role of the SG1 protein identified in previous studies in sporozoite invasion of salivary glands remains to be determined. However, from previous experiments, Saglin was identified using a monoclonal antibody against salivary glands to effectively inhibit P. yoeliisporozoite invasion of Anophelesgambiae salivary glands (Brennan et al., 2000). The Injection of anti-saglin antibody (but not of a control antibody) into the hemocoel of living mosquitoes that strongly inhibits sporozoite invasion (Korochkinaet al., 2006); and Immuno-electronmicrographs (Brennan et al., 2000) are evidence establishing that saglin is present at this location. These results are only compatible with the presence of saglin on the salivary gland surface facing the hemocoel and the saglin gene is predicted to encode a $50 \mathrm{kDa}$ glycoprotein harbouring a signal sequence at the N-terminus of SG1 protein (Okulate, Kalume, Reddy, Kristiansen, \& Bhattacharyya, 2007). There is precedent for surface proteins without transmembrane domains, such as enolase (Bergmann, Rohde, Chhatwa, \& Hammerschmidt, 2001) and the IL-2 receptor (Yeeet al., 2004). However, the mechanisms of surface attachment by such proteins are not well understood. It is thought that glutamines can promote protein-protein interactions through hydrogen bond formation (Stanley \&Fleming, 2007) and it is most probable that the relative high abundance of glutamine residues in saglin perform this function. The resolution of previously published immune-electronmicrographs (Brennan et al., 2000) is not sufficient to determine whether saglin is part of the salivary gland surface or of the basal lamina. There is evidence that other sporozoite proteins also play a role in salivary gland invasion. The synthetic peptide (KLKQP) from the circumsporozoite (CS) protein region I inhibit $P$. Bergheisporozoite salivary gland invasion (Myunget al., 2004) but the receptor of this peptide has not been identified. Although it is shown that parasites harbouring a deletion of CS region II lost their gliding motility and infectivity of the salivary gland (Tewari, Spaccapelo, Bistoni, Holder, \& Cristani, 2000). 


\subsection{Prevention of the Interaction of Surface Enolase and Plasminogen of Mammalian Blood}

Enolase from various microorganisms has a unique 6-AA motif that is recognized by the lysine-binding Kringle domains of plasminogen from mammalian species (Jolodaret al., 2003; Bernal et al., 2004). This motif endows enolase with plasminogen-binding capacity, thus enabling the capture of plasminogen from the surrounding environment. Binding of plasminogen to the lysine motif of enolase is governed by five Kringle domains in the A-chain of plasminogen (Ponting, Marshall, \& Cederholm-Williams, 1992). The Kringle domains of plasminogen bind to the terminal lysine residue of fibrin, triggering its degradation. 6HA (6'-O-phosphoryl-D-arabinohexitol) binds with high affinity to Kringle domains, thus inhibiting plasminogen activation and fibrin degradation (Kupcsik, Alini, \& Stoddart, 2009). Similarly, 6HA had a strong inhibitory effect on oocyst formation, providing additional evidence that plasmin plays an important role in ookinete invasion of the mosquito midgut.Bioinformatics analyses of plasminrevealed that this lysine motif, DKSLVK, is conserved among enolase of several microbial pathogens and is identical in all Plasmodium species. Based on this information, it is predicted that the lysine motif of enolase on the ookinete surface mediates the binding to the mammalian plasminogen present in the ingested blood meal. Immuno-fluorescence assays revealed that plasminogen binds to both $P$. berghei and $P$. Falciparumookinetes. This interaction is specific, as indicated by the competitive inhibition of plasminogen binding by the lysine motif peptide DKSLVK. Control experiments indicated that inhibition of plasminogen binding by the DKSLVK peptide is specific because it did not affect binding of anti-Pbs21(monoclonal antibody 13.1) and anti-enolase antibodies to the ookinete surface. Furthermore, a peptide derivative that had the two lysines replaced by alanines did not inhibit plasminogen binding. The inhibition of plasminogen binding cantruncateplasmodium transmission between the mosquito and the host, a novel exploit for the control of the malaria scourge.

\section{Plants a Natural Competitor for Plasmodium TRAP-Saglin Binding}

Plasmodium TRAP-saglin binding inhibition by SM1 peptide, a promising malaria control method can be further exploited. SM1 is a cystiene rich peptide, cyclic in nature with a disulphide bridge (Ghosh et al., 2009). The disulphide bridge helps to enhance structural stability of the peptide when it attaches itself to membrane receptors of cell. Plants with antimicrobial peptides, which possess structural similarities to SM1 peptide can be consider as a novel competitive inhibitor of plasmodium TRAP-saglin binding.

As part of defence response, plants produce a large number of toxic substances, including antimicrobial peptides (AMPs) (that destroy pathogens via their interactions with phospholipids) and membrane permeabilization proteins (including, cell-penetrating peptides (CPPs)which is capable of introducing into cells a variety of toxicants when specific receptors are lacking by interacting at some point with membrane phospholipids). AMPs and CPPs are part of the nonspecific host defence system and are active against different types of microorganisms (Eudes\&Chugh, 2008; Rivas, Luque-Ortega, Fernandez-Reyes, \& Andreu, 2010; Pelegrini, Quirino\& Franco, 2011; Hegedus \& Marx, 2013). Plants antimicrobial peptides has been isolated from roots, seeds, flowers, stems, and leaves from a large number of species and have shown phytoactivities towards organisms (viruses, bacteria, fungi, protozoa, parasites, and neoplastic cells), that are pathogenic to human, (Montesinos, 2007). They exhibit different kinds of functions, which ranges from direct antimicrobial properties to immune modulatory effects (Choi, Chow, \& Mookherjee,2012). Moreover, AMPs have been found to inactivate prokaryotic cells through the targeting of a number of essential or metabolic processes at extracellular, plasma membrane, and/or intracellular sites (Yount\&Yeaman, 2013). Different plants with antimicrobial peptides exist but the specific antimicrobial peptide which can serve as an analogue of SM1 peptide to competitively inhibit the interaction between plasmodium TRAP and anopheles saglin protein is the cyclotide.

Cyclotides are a group of naturally occurring circular peptides that have been discovered in certain bacteria, plants, and animals (Pelegriniet al., 2007; Craik, 2010). They appear to have high sequence homology with structural identity. Plant cyclotides comprise of 28-37 amino acids, contain a head-to-tail cyclised backbone, and three intra-molecular-disulfide bonds (bridges) arranged in a cysteine backbone knot topology (cyclic cysteine knot, CCK).Below table 1 show common plants containing cyclotides that are commonly used in Nigeria and sSA for the treatment of malaria. 
Table 1. Common plant species with cyclotide peptide

\begin{tabular}{lllll}
\hline S/N & Common Name & Botanical Name & Family Name & Local Names \\
\hline $\mathbf{1}$ & Brimstone tree & Morindalucida (Benth) & Rubiaceae & Oruwo (Yoruba), Eze-ogu (Ibo), Idonzakara (Hausa) \\
$\mathbf{2}$ & Bitter gourd & Momordicacharantia L. & Cucurbitaceae & Ejiinrinw (Yoruba), Alo-ose (Ibo), Kakayi (Hausa) \\
$\mathbf{3}$ & Lemon grass & Cymbopogoncitrates & Poaceae & Koriko-oba (Yoruba), Nch-anwu (Ibo), No name in Hausa \\
$\mathbf{4}$ & Kuntze (P. Beauv) & Rinoreadentate & Violaceae & Ebakwiyiga (Edo), Embeoloborobo (Yoruba), Inimawa (Ijo-Izon) \\
\hline
\end{tabular}

Cyclotides were isolated from the plants belonging to family Violaceae, Rubiaceae, Cucurbitaceae, Poaceae, Asterids, Rosids, and Monocots (Gruber, 2010).The formation of intramolecular disulphide bridges by cyclotides, potentiate its structural similarity and selective inhibitory function to SM1 peptide as, the reduction of the disulphide bond between two cysteine molecules in SMI peptide abolished its binding (Yee et al., 2004). Anti-SM1 antibody recognizes sporozoite TRAP, enhancing the TRAP domain-A, to bind the salivary glands, competed for by the SM1 peptide (Ghoshet al., 2009). This suggested also the usefulness of the disulphide bridge in SM1 peptide and that of the cyclotide peptide.

The cysteine knot is formed by the disulfide bridges between Cys-1-Cys-4 and Cys-2-Cys-5 and their interconnecting backbone form a ring that is penetrated by Cys-3-Cys-6 disulfide bonds (Colgrave\&Craik, 2004).

CCK is largely responsible for the exceptional stability of cyclotides, further suggesting plants containing this antimicrobial peptide can possess antiplasmodial effect similar to SM1 peptide in inhibiting the interaction of TRAP and saglin protein. It is thought such stability of the peptide will help conform it with strong covalent attachment to the saglin protein thereby preventing other proteins like plasmodium TRAP to attach. These stability forces the hydrophobic parts of the protein to be exposed at the molecular surface as the hydrophobic residues form a patch on the surface, making the overall structure amphipathic (Pränting, Loov, Burman, Goransson, \& Andersson, 2010). The presence of this amphipathic structure allows for the binding of proteins with either a net positive charge or net negative charge respectively, with resilient to various proteolytic and degradative processes (Ireland, Clark, Daly, \& Craik, 2010).

The precursor of this peptide contain an endoplasmic reticulum ER signal, an N-terminal repeat (NTR), and a cyclotide sequence domain, followed by a short tail (Craik, 2010). Individual cyclotide genes encode between one and three repeats of the NTR and cyclotide domain to form multiple cyclotide from a single precursor. The gene-encoding pattern of the peptide indicated cyclotide peptide can inhibit the binding of plasmodium TRAP to anopheles saglin protein thereby truncating plasmodium transmission. Plant belonging to the aforementioned families and which possess cyclotide antimicrobial peptide can provide bioactive molecule with antiplasmodial activity.

It is thought that plants containing the antimicrobial peptide "cyclotide" can provide a novel means/therapy for the control of malarial disease by blocking the invasion of ookinete which is believed to be mediated by surface enolase in the mosquito midgut epithelium.

As reviewed above, cyclotide, an antimicrobial peptide isolated from the plants belonging to family Violaceae, Rubiaceae, Cucurbitaceae, and Poaceae (Gruber, 2010) possess structural similarity to SM1 peptide suggesting that it can inhibit the binding of surface enolase to midgut receptor which aids the ookinete invasion. This opened another possible point of target for cyclotide as a novel antiplasmodial agent in the search for bioactive phytochemicals, which can help to fight the dreadful disease (malaria).

\subsection{Blocking Plasmodium Transmission Using Phyto-Active Compounds}

The link between medicinal plants and successful anti-malarial drug discovery dates as far back as 1820.This was the time in, which quinine was isolated from Cinchona bark (Achanet al., 2011). This remain relevant as it continues to the present time, by the development of various potent forms of artemisinin combination therapy (ACT) based on semi-synthetic derivatives of artemisinin, a highly oxygenated sesquiterpene isolated as active compound of Artemisia annua (Weather et al., 2012). The multistage activity of artemisinin derivatives on asexual blood stages and early gametocytes (Pukrittayakameeet al., 2004), has given researchers the idea to explore molecules of plant origin seeking not only activity on parasite stages developing in the vertebrate host, but also transmission blocking effects against the sporogonic stages developing in the mosquito vector. Recently, Lucantoniet al. experimented the in vivo transmission blocking property of azadirachtin-A enriched formulation (NeemAzal) on Plasmodium bergheisporogonic development in Anopheles stephensi mosquitoes (Lucantoniet al., 2010). When NeemAzal was administered to mice at a dose of $50 \mathrm{mg} / \mathrm{kg}$, the commercial Azadirachtaindica seed extract (Kleeberg, 2004) was observed to completely block parasite development in the vector. Azadirachtin A has 
been shown to inhibit the formation of flagellate microgametes from microgametocytes with an IC 50 of $3.5 \mu \mathrm{M}$ (Jones et al., 1994). This suggests that microgametogenesis is a main target process of NeemAzal (azadirachtin-A enriched formulation) transmission blocking action. The transmission blocking activity of this product was recently confirmed in the human parasite P. falciparum by studies conducted in Burkina Faso on field isolates: gametocytaemic blood supplemented with $70 \mathrm{ppm}$ NeemAzal and membrane fed to Anopheles coluzzii mosquitoes completely inhibited oocyst development and therefore infection in mosquitoes (Yerbangaet al., 2014).

In many tropical and sub-Sahara African countries, where malaria is endemic and in particular in rural areas where access to modern health care facilities is often limited, traditional practices still play an important role. People prefer the use of herbal remedies for several reasons including: easier access, lower cost, lack of awareness about modern drugs and belief that the use of traditional medicine is more safe and effective (Adera, 2003). In present day, standardized anti-malarial phytomedicines are officially commercialized in various malaria endemic countries over the world, namely China, Ghana, India, Mali and Burkina Faso. Among few of such products for which clinical research has been conducted, good results were obtained with Qing hao (Artemisia annua, Democratic Republic of Congo trials), Totaquina (Cinchona spp., Multi-country trials) and Phyto-laria (Cryptolepissanguinolenta, Ghana trial) indicating that a parasite clearance at days 5-7 after treatment of 70-100, 92-100 and $100 \%$, respectively. And the clearance of the parasite would mean truncation of the circle. These findings support their use as complementary tools to the conventional anti malarial interventions or as alternative treatments in the absence of anti-malarial drugs (Willcox, 2011). In summary, engagement world wide of the use of Phyto-Active Compounds such as Azadirachtaindica seed extract as above and commercialized standardized anti-malarial phytomedicines already being used in some countries (that enhances parasite clearance) would surely add to the control of malaria.

\section{Suggested Mechanism of Action}

Chemically, phytochemicals with structural similarities to artemisinin, a sesquiterpene lactone containing an unusual peroxide-bridge is thought of to be present in certain plants with medicinal value (Brown, 2006). This peroxide bridge is believed to be responsible for the mechanism of action of artemisinin. Phytochemicals like vernodalin, vernodalol, vernolide, vernomygdalin, 11,13-dihydrovernodalin (present in Vernoniaamygdalina); ascaridole (found in worm seed-oil of Mexican tea) and, sargla peroxide like diterpenes and triterpenes (present in seed of Sarcandraglabra) have been found to possess an unusual peroxide bridge like artemisinin. Due to the structural similarities of the above mentioned phytochemicals to artemisinin, they can be thought of to exhibit similar mode of action in plasmodium transmission blocking. Artemisinin function by its first activation through cleavage after reacting with haem and iron(II) oxide, which results in the generation of free radicals that in turn damage susceptible proteins, resulting in the death of the parasite (Winzeler\&Manary, 2014; Cravo, Napolitano, \& Culleton, 2015). In 2016 artemisinin was shown to bind to a large number of targets suggesting that it acts in a promiscuous manner (Wang et al., 2015). On the basis of the above illustrated broad knowledge available on artemisinin mode of action as an anti-malarial drug-from its evidenced appreciable clinical efficacy to the characterized activity profile of some secondary metabolites as phytochemicals present in certain plants.

Also, nitidine, a product of dihydronitidine oxidation, is the active component in several traditional anti-malarial remedies (Bouquet et al., 2012). The structural similarity between nitidine and dihydronitidine suggests that these compounds share similar mechanism of action. Nitidine was suggested to act by binding to DNA (Noel, Yao, \& Philippe, 2007), via the formation of a 1-1 complex with haem in vitro and it also inhibits $\beta$-haematin formation with the same potency as chloroquine (Bouquet et al., 2012), hence, the postulation that nitidine activity is similar to chloroquine; interfering with haemozoin formation in the food vacuole (Goodman et al., 2016). In all, it seems the mechanism of action of these compounds, also involve the haem-mediated decomposition.

These plants due to their possession of the aforementioned phytochemicals with unusual peroxide bridge with ability to open carbon-ring can serve as a valid candidate for investigation on potential inhibitors of Plasmodium transmission stages.

\section{Conclusion}

Conclusively, the novel methods treated in this work include: The disruption of a cell surface transport protein (FLVCR, that pumps heme out of the cell); Gene silencing-techniques (used to reduce the levels of FLVCR in the mosquito gut); Prevention of the interaction between the plasmodium TRAP and the Anopheles Saglin protein( which aid the malaria parasite invasion of the mosquito salivary gland); Prevention of the Interaction of Surface Enolase and Plasminogen of Mammalian Blood (disrupting an important role in ookinete invasion of the mosquito midgut); the use of Plants with antimicrobial peptides (cyclotide) (that possess structural similarities to SM1 peptide, an inhibitor of plasmodium TRAP-saglin binding); and Blocking Plasmodium Transmission Using 
Phyto-Active Compounds(Azadirachtaindica seed extract observed to completely block parasite development in the vector; all add to the already existing arsenal (use of chemical treated nets, insecticides, antimalaria drugs, clearing of the surrounding environment, etcetera) for the control and treatment of malaria.

\section{References}

Abdukadir, A., Ismail, A. U., Sani, I., Elewechi, O., \& Adamu, Y. K. (2016). Cysteine Protease Inhibitors from Calotropisprocera with Antiplasmodial Potential in Mice. Journal of advances in medicinal and pharmaceutical sciences, 6(3), 1-13.

Achan, J., Talisuna, A. O., Erhart, A., Yeka, A., Tibenderana, J. K., \& Baliraine, F. N. (2011). Quinine, an Old Antimalarial Drug in a Modern World: Role in the Treatment of Malaria. Malaria Journal, 10, 144

Adera, T. (2003). Beliefs and Traditional Treatment of Malaria in KisheSettlement Area, Southwest Ethiopia. Ethiopian Medical Journal, 41(25), 34-17.

Akhouri, R. R., Sharma, A., Malhota, P., \& Amit, S. (2008). Role of Plasmodium falciparum Thrombospondin-Related Anonymous Protein in Host-Cell Interactions. Malaria Journal, 7, 63.

Altruda, F., Poli, V., Restagno, G., \& Silengo, L. (1988).Structure of the Human Hemopexin Gene and Evidence for Intron-mediated Evolution.Journal of Molecular Evolution, 27, 102-108.

Bergmann, S., Rohde, M., Chhatwa, G. S., \& Hammerschmidt, S. (2001). Alpha-Enolase of Streptococcus pneumoniae is a plasminogen-binding protein displayed on the bacterial cell surface. Molecular Microbiology, 40, 1273-1287.

Bernal, D., de la Rubia, J. E., Carrasco-Abad, A. M., Toledo, R., Mas-Coma, S., \& Marcilla, A. (2004). Identification of enolase as a plasminogen-binding protein in excretory- secretory products of Fasciolahepatica. FEBS Lett., 563(1-3), 203-206.

Breman, J. G., Egan, A., \& Keusch, G. T. (2001). The Intolerable Burden of Malaria: a new look at the numbers. American Journal of Tropical Medicine and Hygiene, 64(1-2), iv-vii.

Brennan, J. D., Kent, M., Dhar, R., Fujioka, H., \& Kumar, N. (2000). Anopheles gambiae salivary gland proteins as putative targets for blocking transmission of malaria parasites. Proceedings of the National Academy of Sciences, 97(25), 13859-13864.

Brown, G. D. (2006). Artemisinin and a new generation of antimalarial drugs. Education in Chemistry, 43(4), 97-99.

Choi, K. Y., Chow, L. N., \& Mookherjee, N. (2012). Cationic host defence peptides: multifaceted role in immune modulation and inflammation. Journal of innate immunity, 4(4), 361-370.

Craik, D. J. (2010). Discovery and applications of plant cyclotides. Toxicon, 57, 1092-1102.

Cravo, P., Napolitano, H., \& Culleton, R. (2015). How genomics is contributing to the fight against artemisinin-resistant malaria parasites. ActaTropic, 148, 1-7.

Dana, A. N., Hong, Y. S., Kern, M. K., Hillenmeyer, M. E., Harker, B. W., Lobo, N. F., ... Collins, F. H. (2005). Gene Expression Patterns Associated with Blood- Feedingin the Malaria Mosquito Anopheles gambiae. BMC, Genomics, 6(5).

del Carmen Rodríguez, M., Martínez-Barnetche, J., Alvarado-Delgado, A., Batista, C., Argotte-Ramos, R. S., Hernández-Martínez, S., ... \& Rodríguez, M. H. (2007). The surface protein Pvs25 of Plasmodium vivax ookinetes interacts with calreticulin on the midgut apical surface of the malaria vector Anopheles albimanus. Molecular and biochemical parasitology, 153(2), 167-177.

Dessens, J. T., Sidén-Kiamos, I., Mendoza, J., Mahairaki, V., Khater, E., Vlachou, D., ... \& Sinden, R. E. (2003). SOAP, a novel malaria ookinete protein involved in mosquito midgut invasion and oocyst development. Molecular microbiology, 49(2), 319-329.

Dinglasan, R. R., Kalume, D. E., Kanzok, S. M., Ghosh, A. K., Muratova, O., Pandey, A., \& Jacobs-Lorena, M. (2007). Disruption of Plasmodium falciparum development by antibodies against a conserved mosquito midgut antigen. Proceedings of the National Academy of Sciences, 104(33), 13461-13466.

Eudes, F., \& Chugh, A. (2008). Cell-penetrating peptides: From mammalian to plant cells. Plant signaling \& behavior, 3(8), 549-550. 
Gantt, S., Persson, C., Rose, K., Birkett, A. J., Abagyan, R., \& Nussenzweig, V. (2000). Antibodies against thrombospondin-related anonymous protein do not inhibit Plasmodium sporozoite infectivity in vivo. Infection and immunity, 68(6), 3667-3673.

Ghosh, A. K., \& Jacobs-Lorena, M. (2011). Surface-expressed enolases of Plasmodium and other pathogens. Memorias do Instituto Oswaldo Cruz, 106, 85-90.

Ghosh, A. K., Coppens, I., Gårdsvoll, H., Ploug, M., \& Jacobs-Lorena, M. (2011). Plasmodium ookinetes coopt mammalian plasminogen to invade the mosquito midgut. Proceedings of the National Academy of Sciences, 108(41), 17153-17158.

Ghosh, A. K., Devenport, M., Jethwaney, D., Kalume, D. E., Pandey, A., Anderson, V. E., ... \& Jacobs-Lorena, M. (2009). Malaria parasite invasion of the mosquito salivary gland requires interaction between the Plasmodium TRAP and the Anopheles saglin proteins. PLoS pathogens, 5(1), e1000265.

Ghosh, A. K., Ribolla, P. E., \& Jacobs-Lorena, M. (2001). Targeting Plasmodium ligands on mosquito salivary glands and midgut with a phage display peptide library. Proceedings of the National Academy of Sciences, 98(23), 13278-13281.

Girma, J. P., Meyer, D., Verweij, C. L., Pannekoek, H., \& Sixma, J. J. (1987). Structure-function relationship of human von Willebrand factor. Blood, 70(3), 605-611.

González-Lázaro, M., Dinglasan, R. R., de la Cruz Hernández-Hernández, F., Rodríguez, M. H., Laclaustra, M., Jacobs-Lorena, M., \& Flores-Romo, L. (2009). Anopheles gambiae Croquemort SCRBQ2, expression profile in the mosquito and its potential interaction with the malaria parasite Plasmodium berghei. Insect biochemistry and molecular biology, 39(5-6), 395-402.

Goodman, C. D., Austarheim, I., Mollard, V., Mikolo, B., Malterud, K. E., McFadden, G. I., \& Wangensteen, H. (2016). Natural products from Zanthoxylum heitzii with potent activity against the malaria parasite. Malaria journal, 15(1), 481.

Gruber, C. W. (2010). Global cyclotide adventure: a journey dedicated to the discovery of circular peptides from flowering plants. Peptide Science, 94(5), 565-572.

Hegedüs, N., \& Marx, F. (2013). Antifungal proteins: more than antimicrobials?. Fungal biology reviews, 26(4), $132-145$.

Hood, E. (2004). RNAi: What's all the noise about gene silencing?. Environmental Health Perspectives, 112(4), A224.

Ireland, D. C., Clark, R. J., Daly, N. L., \& Craik, D. J. (2010). Isolation, sequencing, and structure- activity relationships of cyclotides. Journal of natural products, 73(9), 1610-1622.

Ito, J., Ghosh, A., Moreira, L. A., Wimmer, E. A., \& Jacobs-Lorena, M. (2002). Transgenic anopheline mosquitoes impaired in transmission of a malaria parasite. Nature, 417(6887), 452.

Jethwaney, D., Lepore, T., Hassan, S., Mello, K., Rangarajan, R., Jahnen-Dechent, W., ... \& Sultan, A. A. (2005). Fetuin-A, a hepatocyte-specific protein that binds Plasmodium berghei thrombospondin-related adhesive protein: a potential role in infectivity. Infection and immunity, 73(9), 5883-5891.

Jolodar, A., Fischer, P., Bergmann, S., Büttner, D. W., Hammerschmidt, S., \& Brattig, N. W. (2003). Molecular cloning of an $\alpha$-enolase from the human filarial parasite Onchocerca volvulus that binds human plasminogen. Biochimica et Biophysica Acta (BBA)-Gene Structure and Expression, 1627(2-3), 111-120.

Jones, I. W., Denholm, A. A., Ley, S. V., Lovell, H., Wood, A., \& Sinden, R. E. (1994). Sexual development of malaria parasites is inhibited in vitro by the neem extract azadirachtin, and its semi-synthetic analogues. FEMS microbiology letters, 120(3), 267-273.

Kalume, D. E., Okulate, M., Zhong, J., Reddy, R., Suresh, S., Deshpande, N., ... \& Pandey, A. (2005). A proteomic analysis of salivary glands of female Anopheles gambiae mosquito. Proteomics, 5(14), 3765-3777.

Kappe, S., Bruderer, T., Gantt, S., Fujioka, H., Nussenzweig, V., \& Ménard, R. (1999). Conservation of a gliding motility and cell invasion machinery in Apicomplexan parasites. The Journal of cell biology, 147(5), 937-944. 
King, C. L., Adams, J. H., Xianli, J., Grimberg, B. T., McHenry, A. M., Greenberg, L. J., ... \& Zimmerman, P. A. (2011). Fya/Fyb antigen polymorphism in human erythrocyte Duffy antigen affects susceptibility to Plasmodium vivax malaria. Proceedings of the National Academy of Sciences, 108(50), 20113-20118.

Kleeberg, H. (2004). Neem based products: registration requirements, regulatory processes and global implications. In Neem: Today and in the new millennium (pp. 109-123). Springer, Dordrecht.

Korochkina, S., Barreau, C., Pradel, G., Jeffery, E., Li, J., Natarajan, R., ... \& Vernick, K. D. (2006). A mosquito-specific protein family includes candidate receptors for malaria sporozoite invasion of salivary glands. Cellular microbiology, 8(1), 163-175.

Kupcsik, L., Alini, M., \& Stoddart, M. J. (2008). Epsilon-aminocaproic acid is a useful fibrin degradation inhibitor for cartilage tissue engineering. Tissue Engineering Part A, 15(8), 2309-2313.

Lavazec, C., Boudin, C., Lacroix, R., Bonnet, S., Diop, A., Thiberge, S., ... \& Bourgouin, C. (2007). Carboxypeptidases B of Anopheles gambiae as targets for a Plasmodium falciparum transmission-blocking vaccine. Infection and immunity, 75(4), 1635-1642.

Lawler, J., \& Hynes, R. O. (1986). The structure of human thrombospondin, an adhesive glycoprotein with multiple calcium-binding sites and homologies with several different proteins. The Journal of Cell Biology, 103(5), 1635-1648.

Lucantoni, L., Yerbanga, R. S., Lupidi, G., Pasqualini, L., Esposito, F., \& Habluetzel, A. (2010). Transmission blocking activity of a standardized neem (Azadirachta indica) seed extract on the rodent malaria parasite Plasmodium berghei in its vector Anopheles stephensi. Malaria journal, 9(1), 66.

Ma, B., Day, J. P., Phillips, H., Slootsky, B., Tolosano, E., \& Doré, S. (2016). Deletion of the hemopexin or heme oxygenase-2 gene aggravates brain injury following stroma-free hemoglobin-induced intracerebral hemorrhage. Journal of neuroinflammation, 13(1), 26.

Matuschewski, K., Nunes, A. C., Nussenzweig, V., \& Ménard, R. (2002). Plasmodium sporozoite invasion into insect and mammalian cells is directed by the same dual binding system. The EMBO Journal, 21(7), 1597-1606.

Mehta, N. U., \& Reddy, S. T. (2015). Role of hemoglobin/heme scavenger protein hemopexin in atherosclerosis and inflammatory diseases. Current opinion in lipidology, 26(5), 384.

Mocellin, S., \& Provenzano, M. (2004). RNA interference: learning gene knock-down from cell physiology. Journal of translational medicine, 2(1), 39.

Montesinos, E. (2007). Antimicrobial peptides and plant disease control. FEMS microbiology letters, 270(1), $1-11$.

Myung, J. M., Marshall, P., \& Sinnis, P. (2004). The Plasmodium circumsporozoite protein is involved in mosquito salivary gland invasion by sporozoites. Molecular and biochemical parasitology, 133(1), 53-59.

National Center for Biotechnology Information. (n.d.)."Gene Silencing".

Okulate, M. A., Kalume, D. E., Reddy, R., Kristiansen, T., Bhattacharyya, M., Chaerkady, R., ... \& Kumar, N. (2007). Identification and molecular characterization of a novel protein Saglin as a target of monoclonal antibodies affecting salivary gland infectivity of Plasmodium sporozoites. Insect molecular biology, 16(6), 711-722.

Pelegrini, P. B., Quirino, B. F., \& Franco, O. L. (2007). Plant cyclotides: an unusual class of defense compounds. peptides, 28(7), 1475-1481.

Ponting, C. P., Marshall, J. M., \& Cederholm-Williams, S. A. (1992). Plasminogen: a structural review. Blood coagulation \& fibrinolysis: an international journal in haemostasis and thrombosis, 3(5), 605-614.

Pränting, M., Lööv, C., Burman, R., Göransson, U. L. F., \& Andersson, D. I. (2010). The cyclotide cycloviolacin O2 from Viola odorata has potent bactericidal activity against Gram-negative bacteria. Journal of antimicrobial chemotherapy, 65(9), 1964-1971.

Pränting, M., Lööv, C., Burman, R., Göransson, U. L. F., \& Andersson, D. I. (2010). The cyclotide cycloviolacin $\mathrm{O} 2$ from Viola odorata has potent bactericidal activity against Gram-negative bacteria. Journal of antimicrobial chemotherapy, 65(9), 1964-1971.

Quigley, J. (2010). New Approach to Blocking Malaria Transmission Developed. University of Illinois, Chicago

Redberry, G. (2006). Gene silencing: new research. New York: Nova Science Publishers. 
Rivas, L., Luque-Ortega, J. R., Fernandez-Reyes, M., \& Andreu, D. (2010). Membrane-active peptides as anti-infectious agents. Journal of Applied Biomedicine, 8(3), 159-167.

Rogers, W. O., Malik, A., Mellouk, S., Nakamura, K., Rogers, M. D., Szarfman, A., ... \& Hoffman, S. L. (1992). Characterization of Plasmodium falciparum sporozoite surface protein 2. Proceedings of the National Academy of Sciences, 89(19), 9176-9180.

Rosenberg, R. (1985). Inability of Plasmodium knowlesi sporozoites to invade Anopheles freeborni salivary glands. The American journal of tropical medicine and hygiene, 34(4), 687-691.

Saviano, M., Improta, R., Benedetti, E., Carrozzini, B., Cascarano, G. L., Didierjean, C., ... \& Crisma, M. (2004). Benzophenone Photophore Flexibility and Proximity: Molecular and Crystal-State Structure of a BpaContaining Trichogin Dodecapeptide Analogue. ChemBioChem, 5(4), 541-544.

Schleicher, T. R., Yang, J., Freudzon, M., Rembisz, A., Craft, S., Hamilton, M., ... \& Cresswell, P. (2018). A mosquito salivary gland protein partially inhibits Plasmodium sporozoite cell traversal and transmission. Nature communications, 9(1), 2908.

Sinden, R. (2010). A biologist's perspective on malaria vaccine development. Human vaccines, 6(1), 3-11.

Smith, R. C., Vega-Rodríguez, J., \& Jacobs-Lorena, M. (2014). The Plasmodium bottleneck: malaria parasite losses in the mosquito vector. Memórias do Instituto Oswaldo Cruz, 109(5), 644-661.

Snow, R. W., Guerra, C. A., Noor, A. M., Myint, H. Y., \& Hay, S. I. (2005). The global distribution of clinical episodes of Plasmodium falciparum malaria. Nature, 434(7030), 214.

Stanley, A. M., \& Fleming, K. G. (2007). The role of a hydrogen bonding network in the transmembrane $\beta$-barrel OMPLA. Journal of molecular biology, 370(5), 912-924.

Sultan, A. A., Thathy, V., Frevert, U., Robson, K. J., Crisanti, A., Nussenzweig, V., ... \& Ménard, R. (1997). TRAP is necessary for gliding motility and infectivity of Plasmodium sporozoites. Cell, 90(3), 511-522.

Templeton, T. J., Kaslow, D. C., \& Fidock, D. A. (2000). Developmental arrest of the human malaria parasite Plasmodium falciparum within the mosquito midgut via CTRP gene disruption. Molecular microbiology, 36(1), 1-9.

Tewari, R., Spaccapelo, R., Bistoni, F., Holder, A. A., \& Crisanti, A. (2002). Function of Region I and II Adhesive Motifs ofPlasmodium falciparum Circumsporozoite Protein in Sporozoite Motility and Infectivity. Journal of Biological Chemistry, 277(49), 47613-47618.

Tsai, Y. L., Hayward, R. E., Langer, R. C., Fidock, D. A., \& Vinetz, J. M. (2001). Disruption of Plasmodium falciparumChitinase Markedly Impairs Parasite Invasion of Mosquito Midgut. Infection and immunity, 69(6), 4048-4054.

Wang, J., Zhang, C. J., Chia, W. N., Loh, C. C., Li, Z., Lee, Y. M., ... \& Liew, C. X. (2015). Haem-activated promiscuous targeting of artemisinin in Plasmodium falciparum. Nature communications, 6, 10111.

Wang, S., \& Jacobs-Lorena, M. (2013). Genetic approaches to interfere with malaria transmission by vector mosquitoes. Trends in biotechnology, 31(3), 185-193.

Weathers, P. J., Arsenault, P. R., Covello, P. S., McMickle, A., Teoh, K. H., \& Reed, D. W. (2011). Artemisinin production in Artemisia annua: studies in planta and results of a novel delivery method for treating malaria and other neglected diseases. Phytochemistry Reviews, 10(2), 173-183.

Willcox, M. (2011). Improved traditional phytomedcines in current use for the clinical treatment of malaria. Plants Medicine, 77, 662-671.

World Health Organization. (2017). The World Malaria Report.

Yang, Z., Philip, J. D., Raymond, T. D., Pablo, G., Ostrow, J. D., Tiribelli, C., Smith, A., \& Abkowitz, J. I. (2010). Kinetic and Specificity of FLVCR Export Function and its Dependence on Hemopexin. Journal of Biology. http://www.jbc.org/cgi/doi/10.1074/jbc.M110.119131.

Yang, Z., Philips, J. D., Doty, R. T., Giraudi, P., Ostrow, J. D., Tiribelli, C., ... \& Abkowitz, J. L. (2010). Kinetics and specificity of FLVCR export function and its dependence on hemopexin. Journal of Biological Chemistry, jbc-M110.

Yee, Y., Shibata, Y., Yun, C., Ron, D., \& Rapoport, T. A. (2004). A membrane protein complex mediates retro-translocation from the ER lumen into the cytosol. Nature, 429, 841-847. 
Yerbanga, R., Lucantoni, L., Ouédraogo, R., Da, D. F., Yaméogo, K., \& Churcher, T. (2014). Transmission blocking activity of Azadirachtaindica and Guierasenegalensis extracts on the sporogonic development of Plasmodium falciparum field isolates in Anopheles coluzzii mosquitoes. Parasites and Vectors, 7, 185.

Yount, N. Y., \& Yeaman, M. R. (2013). Peptide antimicrobials: cell wall as a bacterial target. Annual, New York Academic Science, 1277, 127-138.

\section{Copyrights}

Copyright for this article is retained by the author(s), with first publication rights granted to the journal.

This is an open-access article distributed under the terms and conditions of the Creative Commons Attribution license (http://creativecommons.org/licenses/by/4.0/). 